\title{
Gravity, Cosmology and Particle Physics without the Cosmological Constant Problem"
}

\author{
E.I.Guendelman'] and A.B.Kaganovich \\ Physics Department, Ben Gurion University, Beer Sheva, Israel
}

\begin{abstract}
This essay elucidates recent achievements of the "nongravitating vacuum energy" (NGVE) theory" which has the feature that a shift of the Lagrangian density by a constant does not affect dynamics. In the first order formalism, a constraint appears that enforces the vanishing of the cosmological constant $\Lambda$. Standard dynamics of gauge unified theories (including fermions) and their SSB appear if a four index field strength condensate is present. At a vacuum state there is exact balance to zero of the gauge fields condensate and the original scalar fields potential. As a result it is possible to combine the solution of the $\Lambda$ problem with inflation and transition to a $\Lambda=0$ phase without fine tuning after a reheating period. The model opens new possibilities for a solution of the hierarchy problem.
\end{abstract}

\footnotetext{
${ }^{1}$ This essay received an "honorable mention" from the Gravity Research Foundation, 1998.

${ }^{2}$ Electronic address: GUENDEL@BGUmail.BGU.AC.IL

${ }^{3}$ Electronic address: ALEXK@BGUmail.BGU.AC.IL
} 
A familiar notion in theoretical physics is that the "origin of energy is not important". Gravity, if formulated in the standard way opposes such idea: in General Relativity (GR) the action $\int L \sqrt{-g} d^{4} x$ is not invariant under the shift $L \rightarrow L+$ const, which in fact gives rise to a cosmological constant in the equations of motion. Here we want to restore $L \rightarrow L+$ const as a symmetry, even if gravity is introduced. For this we change $\sqrt{-g} d^{4} x$ by the volume form $d \varphi_{1} \wedge d \varphi_{2} \wedge d \varphi_{3} \wedge d \varphi_{4} \equiv \frac{\Phi}{4 !} d^{4} x$ where $\Phi \equiv \varepsilon_{a b c d} \varepsilon^{\mu \nu \alpha \beta}\left(\partial_{\mu} \varphi_{a}\right)\left(\partial_{\nu} \varphi_{b}\right)\left(\partial_{\alpha} \varphi_{c}\right)\left(\partial_{\beta} \varphi_{d}\right)$, and $\varphi_{a} \quad(a=1, \ldots, 4)$, the measure fields, are independent degrees of freedom. Since $\Phi$ is a total divergence, the symmetry $L \rightarrow L+$ const, i.e. the NGVE principle, is automatically satisfied [1].

Having allowed the measure not to be necessarily $\sqrt{-g}$, but to be determined dynamically, it seems natural also to allow the connection to be determined dynamically and not to assume it to be the Christoffel symbol from the beginning. This is the case in the first order formalism, which leads to the resolution of the cosmological constant problem in the NGVE theory [2]-[3].

We consider then the action $S=\int \Phi L d^{4} x$ and assume that $L$ does not contain the measure fields, that is the fields by means of which $\Phi$ is defined. If this condition is satisfied then the theory has an additional symmetry [1]. Our choice for the total Lagrangian density is $L=\kappa^{-1} R(\Gamma, g)+L_{m}$, where $L_{m}$ is the matter Lagrangian density and $R(\Gamma, g)$ is the scalar curvature $R(\Gamma, g)=g^{\mu \nu} R_{\mu \nu}(\Gamma)$ of the space-time of the affine connection $\Gamma_{\alpha \beta}^{\mu}: \quad R_{\mu \nu}(\Gamma)=R_{\mu \nu \alpha}^{\alpha}(\Gamma), \quad R_{\mu \nu \sigma}^{\lambda}(\Gamma) \equiv \Gamma_{\mu \nu, \sigma}^{\lambda}-\Gamma_{\mu \sigma, \nu}^{\lambda}+$ $\Gamma_{\alpha \sigma}^{\lambda} \Gamma_{\mu \nu}^{\alpha}-\Gamma_{\alpha \nu}^{\lambda} \Gamma_{\mu \sigma}^{\alpha}$. This curvature tensor is invariant under the $\lambda$ - transformation [5] $\Gamma_{\alpha \beta}^{\prime \mu}=\Gamma_{\alpha \beta}^{\mu}+\delta_{\alpha}^{\mu} \lambda_{, \beta}$. In the NGVE-theory, it allows us to eliminate the contribution 
to the torsion which appears as a result of introduction of the new measure. However, even after this still there is the non metric contribution to the connection related to the measure (it is expressed in terms of derivatives the scalar field $\chi \equiv \Phi / \sqrt{-g}$ ).

In addition to this, in the vacuum and in some matter models, the theory possesses a local symmetry which plays a major role. This symmetry consists of a conformal transformation of the metric $g_{\mu \nu}(x)=J^{-1}(x) g_{\mu \nu}^{\prime}(x)$ accompanied by a corresponding diffeomorphism $\varphi_{a} \longrightarrow \varphi_{a}^{\prime}=\varphi_{a}^{\prime}\left(\varphi_{b}\right)$ in the space of the scalar fields $\varphi_{a}$ such that $J=\operatorname{Det}\left(\frac{\partial \varphi_{a}^{\prime}}{\partial \varphi_{b}}\right)$. Then for $\Phi$ we have: $\Phi(x)=J^{-1}(x) \Phi^{\prime}(x)$. In the presence of fermions this symmetry is appropriately generalized [2]. For models where it holds, it is possible to choose the gauge where the measure $\Phi$ coincides with $\sqrt{-g}$, the measure of GR. This is why we call this symmetry "local Einstein symmetry" (LES).

Varying the action with respect to $\varphi_{a}$ we get $A_{b}^{\mu} \partial_{\mu}\left[-\frac{1}{\kappa} R(\Gamma, g)+L_{m}\right]=0$ where $A_{b}^{\mu}=\varepsilon_{a c d b} \varepsilon^{\alpha \beta \gamma \mu}\left(\partial_{\alpha} \varphi_{a}\right)\left(\partial_{\beta} \varphi_{c}\right)\left(\partial_{\gamma} \varphi_{d}\right)$. If $\operatorname{Det}\left(A_{b}^{\mu}\right)=\frac{4^{-4}}{4 !} \Phi^{3} \neq 0$ then

$$
-\frac{1}{\kappa} R(\Gamma, g)+L_{m}=M=\text { const }
$$

Performing the variation with respect to $g^{\mu \nu}$ we get (here for simplicity we don't consider fermions)

$$
-\frac{1}{\kappa} R_{\mu \nu}(\Gamma)+\frac{\partial L}{\partial g^{\mu \nu}}=0
$$

Contracting eq.(2) with $g^{\mu \nu}$ and making use eq.(1) we get the constraint

$$
g^{\mu \nu} \frac{\partial\left(L_{m}-M\right)}{\partial g^{\mu \nu}}-\left(L_{m}-M\right)=0
$$

For the cases where the LES is an exact symmetry, we can eliminate the mentioned above $\chi$-contribution to the connection. Indeed, for $J=\chi$ we get $\chi^{\prime} \equiv 1$ and 
$\Gamma_{\mu \nu}^{\alpha}=\left\{\begin{array}{l}\alpha \\ \mu \nu\end{array}\right\}^{\prime}$, where $\left\{\begin{array}{l}\alpha \\ \mu \nu\end{array}\right\}^{\prime}$ are the Christoffel's coefficients corresponding to the new metric $g_{\mu \nu}^{\prime}$. In this gauge the affine space-time becomes a Riemannian space-time.

When applying the theory to the matter model of a single scalar field with a potential $V(\varphi)$, the constraint (3) implies $V(\varphi)+M=0$, which means that $\varphi$ is a constant (the equation of motion of $\varphi$ implies also that $V^{\prime}(\varphi)=0$ ). Since $\varphi=$ constant, Eq. (2) implies $R_{\mu \nu}(\Gamma, g)=0$ and also Eq. (11) implies $R(\Gamma, g)=0$, if $V(\phi)+M=0$ is taken into account. $V(\phi)+M=0$ dictates the vanishing of the LES violating terms and disappearance of dynamics of $\varphi$, so LES is effectively restored on the mass shell. Choosing the gauge $\chi=1$ we see that the potential does not have a gravitational effect since the standard Ricci tensor vanishes and flat space-time remains the only natural vacuum.

The above solution of the cosmological constant problem is at the prize of the elimination of a possible scalar field dynamics. We will see now that the introduction of a 4-index field strength can restore normal scalar, gauge and fermion dynamics and as a bonus provide [3], [4]: (i) the possibility of inflation in the early universe with a transition (after reheating) to a $\Lambda=0$ phase without fine tuning and (ii) a solution to the hierarchy problem in the context of unified gauge dynamics with SSB.

A four index field strength is derived from a three index gauge potential according to $F_{\mu \nu \alpha \beta}=\partial_{[\mu} A_{\nu \alpha \beta]}$. The physical scenario we have in mind is one where all gauge fields, including $A_{\nu \alpha \beta}$ are treated in a unified way. The possible physical origin of $A_{\nu \alpha \beta}$ can be for example an effective way to describe the condensation of a vector gauge field in some extra dimensions [6]. In this picture for example this means that all gauge 
fields should appear in a combination having all the same homogeneity properties with respect to conformal transformations of the metric. This is achieved if all dependence on field strengths is through the "gauge fields complex" $y=F_{\mu \nu}^{a} F^{a \mu \nu}+\frac{\varepsilon^{\mu \nu \alpha \beta}}{\sqrt{-g}} \partial_{\mu} A_{\nu \alpha \beta}$.

Considering for illustration only one vector gauge field $\tilde{A}_{\mu}$ and a charged scalar field $\phi$, we take a generic action satisfying the above requirements in the unitary

$$
\begin{aligned}
& \text { gauge }\left(\phi=\phi^{*} ;|\phi|=\frac{1}{\sqrt{2}} \varphi\right) \\
& \qquad S=\int \Phi d^{4} x\left[-\frac{1}{\kappa} R(\Gamma, g)-m^{4} f(u)+\frac{1}{2} g^{\mu \nu} \partial_{\mu} \varphi \partial_{\nu} \varphi-V(\varphi)+\frac{1}{2} \tilde{e}^{2} \varphi^{2} g^{\mu \nu} \tilde{A}_{\mu} \tilde{A}_{\nu}\right]
\end{aligned}
$$

where $u \equiv y / m^{4}, m$ is a mass parameter and $f(u)$ is a nonspecified function which has to have an extremum at some point $u=u_{0}>0$ to provide physically reasonable consequences (see below).

The equations of motion obtained from variating $A_{\nu \alpha \beta}$ imply $\chi f^{\prime}=\omega=$ constant where $\omega$ is a dimensionless integration constant. The constraint (34) becomes now

$$
-2 u f^{\prime}(u)+f(u)+\frac{1}{m^{4}}[V(\varphi)+M]=0,
$$

which allows to find $u=u(\varphi)$.

One can then see that all equations can be put in the standard GR, scalar field and gauge field form if we make the conformal transformation to the "Einstein frame" $\bar{g}_{\mu \nu}=\chi g_{\mu \nu}$. In the Einstein frame, the scalar field acquires an effective potential

$$
V_{e f f}(\varphi)=\frac{y}{\omega}\left(f^{\prime}(u)\right)^{2}
$$

If there is a point $u=u_{0}$ where $f^{\prime}\left(u_{0}\right)=0$, then if $y_{0} / \omega>0$, such a state is a stable vacuum of the theory. This vacuum state is defined by the gauge and 
scalar condensates $\left(u_{0}, \varphi_{0}\right)$ connected by the relation $f\left(u_{0}\right)+\frac{1}{m^{4}}\left[V\left(\varphi_{0}\right)+M\right]=0$, representing the exact cancellation of the contributions to the vacuum energy of the scalar field and of gauge field condensate. Therefore the effective cosmological constant in this vacuum becomes zero without fine tuning.

One can see also that $\frac{d V_{\text {eff }}}{d \varphi}=\frac{1}{\omega} \frac{d f}{d u} \frac{d V}{d \varphi}$, so another extremum where $V^{\prime}=0$ for example can serve as a phase with nonzero effective $\Lambda$ and therefore inflation becomes possible as well. This vacuum is smoothly connected by dynamical evolution of the scalar field, with $\Lambda=0$ one, thus providing a way to achieve inflation and transition (after standard reheating period) to $\Lambda=0$ phase without fine tuning.

Stability of gauge fields requires $\omega>0$. In this case theory acquires canonical form if the new fields and couplings are defined $A_{\mu}=2 \sqrt{\omega} \tilde{A}_{\mu}, \quad e=\frac{\tilde{e}}{2 \sqrt{\omega}}$. Appearance of the VEV of the scalar field $\varphi_{0}$ leads to the standard Higgs mechanism.

Fermions can also be introduced [4] in such a way that normal massive propagation is obtained in the Einstein frame. The resulting fermion mass is proportional to $\frac{\varphi_{0}}{\sqrt{u_{0} \omega}}$. The gauge boson mass, depending on $e$ also goes as $\propto 1 / \sqrt{\omega}$. So we see that a big value of the integration constant $\omega$ pushes both effective masses and gauge coupling constants to small values, thus providing a new approach to the solution of the hierarchy problem. Furthermore we see that fermion masses include additional factor $u_{0}^{-1 / 2}$. Therefore, if the gauge complex condensate $u_{0}$ is big enough, it can explain why fermion masses are much less then boson ones.

Finally, there is no obstacle for the construction of a realistic unified theories (like electroweak, QCD, GUT) along the lines of the simple example displayed above [田. 
The common feature of such theories is the fact that the stable vacuum developed after SSB has zero effective cosmological constant.

\section{References}

[1] E.I. Guendelman and A.B. Kaganovich, Phys. Rev. D53, 7020 (1996).

[2] E.I. Guendelman and A.B. Kaganovich, Phys. Rev.D55, 5970 (1997); ibid., D56, 3548 (1997).

[3] E.I. Guendelman and A.B. Kaganovich, "From inflation to a zero cosmological phase without fine tuning", gr-qc/9709059, to appear in Phys. Rev.D.

[4] E.I. Guendelman and A.B. Kaganovich, "Gauge Unified Theories without the Cosmological Constant Problem", hep-th/9803134.

[5] A.Einstein, The Meaning of Relativity, Fifth Edition, MJF Books, N.Y.1956 ( see Appendix II).

[6] E.I. Guendelman, Phys. Lett. B412, 42 (1997). 